\title{
Emergence of the bifurcation structure of a Langmuir-Blodgett transfer model
}

\author{
Michael H. Köpf \\ Département de Physique, École Normale Supérieure, \\ CNRS, 24 rue Lhomond, 75005 Paris, France* \\ Uwe Thiele \\ Institut für Theoretische Physik, Westfälische Wilhelms-Universität Münster, \\ Wilhelm Klemm Str. 9, D-48149 Münster, Germany ${ }^{\dagger}$
}

\begin{abstract}
We explore the bifurcation structure of a modified Cahn-Hilliard equation that describes a system that may undergo a first order phase transition and is kept permanently out of equilibrium by a lateral driving. This forms a simple model, e.g., for the deposition of stripe patterns of different phases of surfactant molecules through Langmuir-Blodgett transfer. Employing continuation techniques the bifurcation structure is numerically investigated employing the non-dimensional transfer velocity as the main control parameter. It is found that the snaking structure of steady fronts states is intertwined with a large number of branches of time-periodic solutions that emerge from Hopf or period doubling bifurcations and end in global bifurcations (sniper and homoclinic). Overall the bifurcation diagram has a harp-like appearance. This is complemented by a two-parameter study (in non-dimensional transfer velocity and domain size) that elucidates through which local and global codimension 2 bifurcations the entire harp-like structure emerges.
\end{abstract}

*Electronic address: michael.koepf@ens.fr; URL: http://www. michaelkoepf.de

†Electronic address: u.thiele@lboro.ac.uk; URL: http: / /www.uwethiele.de 


\section{INTRODUCTION}

Pattern formation that occurs at moving three-phase contact lines, e.g., where a liquid recedes or advances on a solid substrate under a gas atmosphere, poses significant challenges to both, the experimentalist and the theoretician. A prominent example are deposition patterns, like a ringshaped coffee stain [1], that result from a dynamic process that involves hydrodynamic flow of a solution or suspension, the dynamics of the contact line and evaporation of the solvent $[2,3]$. The problem is significantly enriched by a surface activity of the solute, i.e., by the presence of surfactants, as then Marangoni forces resulting from spatially inhomogeneous surface tensions are induced by concentration gradients [4]. Although, the creation or avoidance of deposition patterns is of high practical relevance [2] and experiments show that a large number of systems produce a wide variety of patterns [3], the understanding and control of this entire class of pattern formation processes is not yet well developed. Here we discuss a reduced model for the deposition of line patterns of surfactant molecules on a plate that is withdrawn from a water-filled trough covered by a floating surfactant monolayer and numerically analyse its solution and bifurcation behaviour. This shall allow us to better understand how the emergence of deposition patterns in out-of-equilibrium situations is related to equilibrium phase transitions that occur in the same system when considered without driving.

A frequently used mathematical framework for problems where the evolution of a free surface of a film or shallow droplet on a substrate needs to be described is the long-wave (or lubrication) approximation [5]. Assuming that all relevant fields have small gradients parallel to the solid substrate the governing hydrodynamic transport equations and boundary conditions are asymptotically reduced to a single (for single layers of simple liquids [5-8]) or coupled (for two or more layers of simple liquids, or various complex liquids [4, 9-11]) fourth order, strongly nonlinear partial differential equations that describe the evolution of film thickness profile(s) and pertinent concentration field(s). Up to now only a few works investigate the deposition of regular one-dimensional line patterns with such long-wave models [12-15] - mainly through direct time-simulations of the derived long-wave evolution equations. Although this allows one to determine parameter regions where line patterns may be deposited, the technique is less than ideal when it comes to understanding the onset of patterning: The time-simulation results support several hypotheses regarding the role of local and global bifurcations in the patterning process (cf., in particular, section 3.4 of Ref. [14] and the concluding paragraph of [15]) but they provide at best an incomplete picture of 
the bifurcation structure, in particular as they are not able to reveal unstable solutions that might coexist with the stable solutions that correspond to the deposition. A deeper analysis is asked for, if one wants to clearly identify the involved bifurcation types and to understand the entire underlying bifurcation structure.

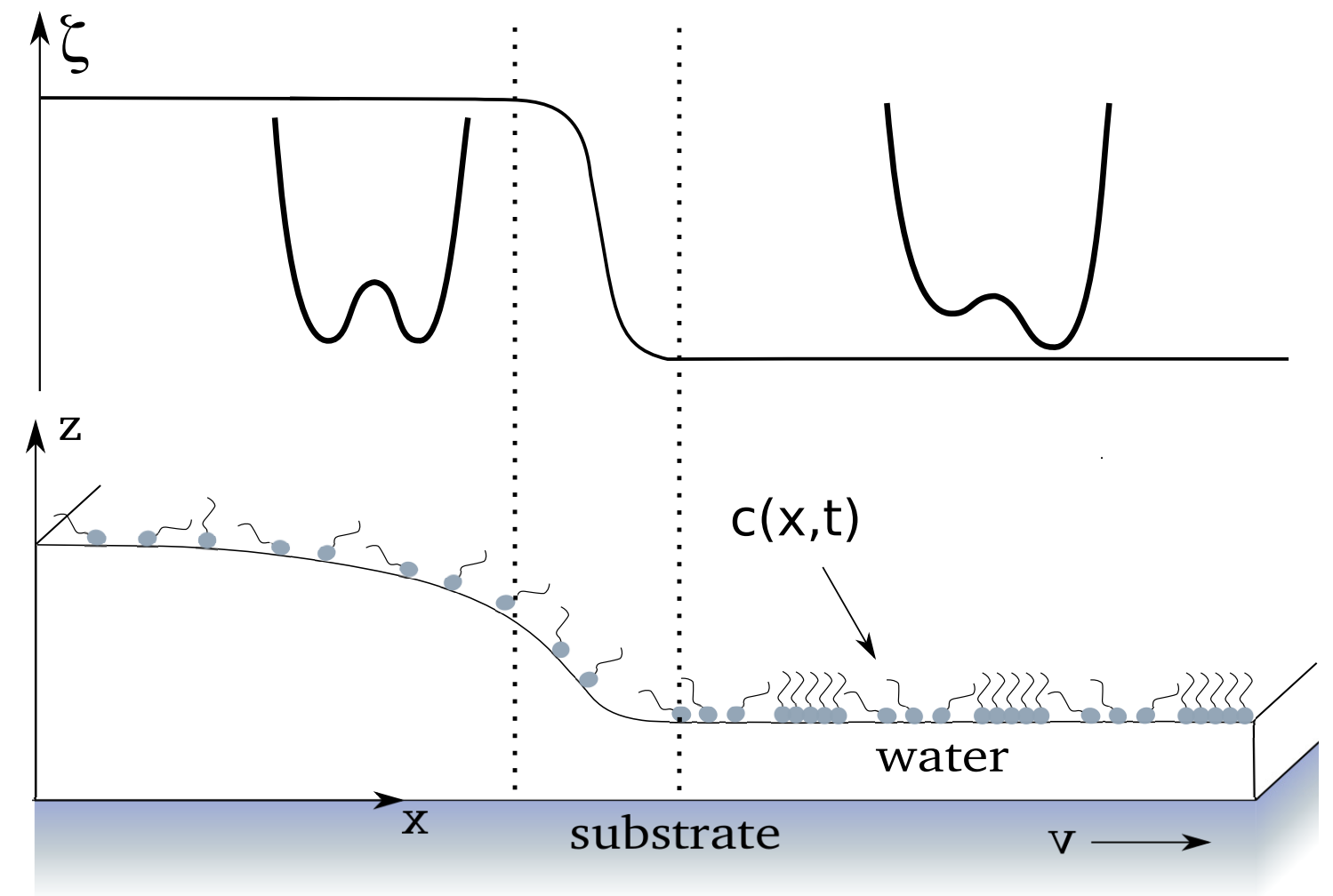

FIG. 1: Schematic drawing of a surfactant-covered meniscus on a solid substrate during the transfer with velocity $v$. The surfactant density at the surface is denoted by $c(x, t)$. The function $\zeta(x)$ describes the change of the tilt of the free energy from the symmetric situation far away from the substrate (inset double well on the left) to the situation where the condensed state is energetically favoured (asymmetric double well on the right).)

Here, we focus on the Langmuir-Blodgett transfer of an insoluble monolayer of surfactant from the surface of a water bath onto a plate that is drawn out of the bath as sketched in Fig. 1. If one uses a suitable amphiphilic molecule as surfactant at conditions sufficiently close to a first order structural liquid-liquid phase transition (liquid-expanded to liquid-condensed, see note [16]) then the intermolecular interaction with the solid plate may trigger a phase transition in parts of the surfactant layer due to an effect called substrate-mediated condensation [17]. On a plate that moves out of a bath, the partial condensation results in rather regular stripe patterns. They 
are preferentially oriented either parallel or perpendicular to the contact line. The wavelength of the resulting pattern depends on the plate velocity and temperature and can range from several micrometers down to a few hundred nanometers [18-20].

For this system there exists a full long-wave description in terms of coupled evolution equations for the film height profile and the surfactant concentration $[12,21]$ as well as a reduced model in terms of a Cahn-Hilliard type evolution equation for a density with amendments to take substratemediated condensation into account [22]. Although the reduced model is a significantly simplified version of the full model, it captures all essential properties including the instabilities leading to stripe deposition parallel and perpendicular to the contact line and even reproduces the results of the full model in the case of structured substrates, where synchronisation effects can be observed $[23,24]$. Most importantly, however, it allowed for a first analysis of the bifurcations related to the onset of pattern formation in the system. A preliminary bifurcation diagram has been obtained (see Fig. 2 below) by a combination of numerical path continuation of steady states that correspond to homogeneous deposition and direct numerical time simulation of time-periodic solutions that correspond to the experimentally observed deposition of regular line patterns.

It was found that a branch of stable time-periodic solutions exists for a range of plate velocities $V$. It ends at high $V$ in a Hopf bifurcation and at low $V$ in a homoclinic bifurcation. Furthermore, it was possible to reveal a structure of the bifurcation diagram in the range of low velocities which is best described by the term front snaking (see Fig. 2 below). The branch of steady solutions snakes back and forth, as more and more bumps are added (one per wiggle) to a localized structure in the transition region (i.e., front) between the bath surface and the drawn-out film. As a result, several (stable or unstable) steady solutions can exist at the same transfer velocity. This behaviour is reminiscent of homoclinic snaking well known from the investigation of other model equations of pattern formation, such as the standard Swift-Hohenberg equation [25-27] and the conserved Swift-Hohenberg equation [28] (often called phase-field crystal equation [29]). However, as further explained below, in our case, the solutions on the snaking curve neither represent homoclinic nor heteroclinic orbits, but can be seen as different front states.

The numerical approach of Ref. [22] was limited w.r.t. the onset of patterning. It only permitted to find the loci of Hopf bifurcations on the branch of steady solutions, but could not be employed to determine the emerging branches of time-periodic solutions from start to end. Therefore, important questions regarding the onset of line pattern formation remain unanswered. The present work overcomes these limitations employing numerical continuation of stable and unstable branches of 
steady states (as in Ref. [22]) and of time-periodic solutions. The resulting completed bifurcation diagram in terms of our primary control parameter - the plate velocity $V$ - is complemented by a study of its dependence on a second dimensionless control parameter that represents the ratio of the domain size to the typical patterning length, i.e., is inverse to the 'undercooling' or quench strength. The resulting two parameter study allows us to start to understand through which codimension 2 local and global bifurcations the intertwined structure of snaking steady states and time-periodic solutions emerges.

The paper is structured as follows. Section II briefly reviews the geometric set-up and model equation of Ref. [22] that is employed here without change, followed by a presentation of the employed numerical method. Section III presents our results while section IV concludes by discussing implications for systems that show similar transitions and by pointing out further open questions and challenges.

\section{MODEL AND NUMERICAL TREATMENT}

\section{A. Evolution equation}

The Langmuir-Blodgett transfer of surfactant monolayers close to a first-order phase transition of the surfactant layer can lead to regular stripe patterns. These stripes correspond to alternating domains of different thermodynamic surfactant phases: liquid-expanded phase (LE) and the liquid-condensed phase (LC) that can be seen as respective analogues of the gas phase and the liquid phase in a standard liquid-gas phase transition of a simple liquid. The dynamics of the stripe formation can be modelled through a long-wave approach that couples the sub-phase hydrodynamics to the nonequilibrium thermodynamics of the monolayer via the surfactant equation of state. In the vicinity of a first-order phase transition, this equation can be derived from a free energy functional of the type proposed by Cahn and Hilliard [30] and determines the dependence of the surface tension of the liquid-air interface on the monolayer density, resulting in a Marangoni force and variations of the Laplace pressure [12, 21, 31]. An analysis of the results of Ref. [12] shows that the full long-wave model may be simplified [22] and the essence of the LE-LC domain formation under the influence of substrate-mediated condensation can be captured by an amended Cahn-Hilliard-type model. The resulting evolution equation consists of the generic Cahn-Hilliard equation describing the dynamics of phase decomposition [32] augmented by the addition of a 
space-dependent external field and a dragging term. The external field models the interaction between the monolayer and the substrate that changes in a step-like manner in the contact line region. The drag term describes that the contact line region where the bath meets the moving plate recedes with nearly constant speed in the reference frame of the plate.

We focus on the situation where the contact line is straight and the plate is drawn in the direction orthogonal to the contact line. In this setting the evolution of the scalar field $c(x, t)$ that represents the concentration of surfactant on the surface of the liquid is captured by the non-dimensionalised evolution equation [22]

$$
\partial_{t} c=\partial_{x}\left[\partial_{x} \frac{\delta F}{\delta c}-V c\right]
$$

where $x$ is the spatial coordinate orthogonal to the contact line (see Fig. 1), $t$ is time, and $V$ is the speed at which the substrate is withdrawn. The substrate dependent interaction field that causes substrate-mediated condensation enters through an explicite space-dependent term $\zeta(x)$ in the free energy density

$$
f(c, x)=\frac{1}{2}\left(\partial_{x} c\right)^{2}-\frac{c^{2}}{2}+\frac{c^{4}}{4}+\mu \zeta(x) c .
$$

It furthermore contains a gradient contribution that penalizes interfaces and a double-well potential. One may say that $\zeta(x)$ is responsible for a space-dependent tilt of the double-well potential.

The free energy density is integrated to obtain the total free energy $F=\int \mathrm{d} x f(c, x)$. We insert $F$ into Eq. (1) and obtain the evolution equation

$$
\partial_{t} c=-\partial_{x}^{2}\left[\partial_{x}^{2} c-c^{3}+c-\mu \zeta(x)\right]-V \partial_{x} c
$$

Note that due to the space-dependence of $f$, Eq. (3) is not invariant with respect to translations in $x$. Therefore one is not able to remove the advection term $V \partial_{x} c$ by a simple Galilei transform. With other words, the spatial dependence selects a particular frame of reference: the physical laboratory system.

The function $\zeta$ smoothly switches between two thermodynamically different regions: (i) left of the contact line ( $x<x_{s}$, where $x_{s}$ is the contact line position) the local free energy is a symmetric double well potential, i.e. the liquid expanded (LE) and liquid condensed (LC) phases are energetically equivalent, and (ii) right of the contact line $\left(x>x_{s}\right)$ the local free energy is tilted favouring the LC phase. As the results are not very sensitive to the particular form of $\zeta$ we use the same hyperbolic tangent centered at $x=x_{s}$ as in Ref. [22]:

$$
\zeta(x)=-\frac{1}{2}\left[1+\tanh \left(\frac{x-x_{s}}{l_{s}}\right)\right] .
$$


Thus, the width of the transition region is determined by the constant $l_{s}$. For consistency with Ref. [22] we use the boundary conditions (BC)

$$
c(0)=c_{0}, \quad \partial_{x x} c(0)=\partial_{x} c(L)=\partial_{x x} c(L)=0 .
$$

The density $c_{0}$ that is imposed at the left boundary of the system models the presence of a virtually non-depletable surfactant bath. In fact, in the experiments, the Langmuir trough is operated in a way that it keeps the surfactant surface pressure, and therefore the density, constant by displacing barriers at the surface of the trough.

Finally we mention a number of related models. Krekhov [33], and Foard and Wagner [34, 35] use related Cahn-Hilliard-type models. In particular, they employ space dependent free energies that are switched from single-well potentials to symmetric double-well potentials at a moving threshold, by switching the sign of the quadratic term of the free energy. Such a threshold models a quench front, dividing the spatial domain into a two-phase and a one-phase region. This differs from the situation considered here, as in our model the threshold divides two different two-phase regions, namely, the region near the boundary on the mensicus side where the free energy is a symmetric double well and the region where the double well is tilted. The state in the region with the symmetric double well is determined by the boundary condition. The quench front investigated in Refs. [33-35] propagates into a one-phase region where the order parameter is spatially uniform. Thus, there is a simple relation between the front velocity and the flow of material to the quench front: If the value of the order parameter in this region is called $c^{\star}$, then the flow of material to the front is $c^{\star} V$ and remains constant with time. In contrast to this situation, the inflow of surfactant in our model is not constant but dynamically adjusts to keep the surfactant density at the left boundary constant. This reflects the physical reality of Langmuir-Blodgett transfer, where the surfactant influx is determined by the hydrodynamic flow field and the dynamics of the surfactant molecules at the water surface, which in turn are influenced by the Langmuir trough that keeps the surfactant density constant in the bath.

\section{B. Non-dimensionalisation}

Although for consistency, we use throughout this article the non-dimensional equation (3) and non-dimensional parameter as in Ref. [22], to understand the physical role of the parameters it is instructive to review the underlying scaling. We indicate dimensional quantities by a tilde and 
write the dimensional equivalent of Eq. (3) as

$$
\partial_{\tilde{t}} \tilde{c}=-M \partial_{\tilde{x} \tilde{x}}\left[\sigma \partial_{\tilde{x} \tilde{x}} \tilde{c}+\kappa\left(a \tilde{c}-\tilde{c}^{3}\right)-\tilde{\mu} \tilde{\zeta}(\tilde{x})\right]-\tilde{V} \partial_{\tilde{x}} \tilde{c}
$$

where $M$ is a diffusional mobility, $\sigma$ is related to interface tension, $\kappa$ is an energy density scale, $\tilde{\mu}$ the chemical potential difference and $\tilde{V}$ is the plate velocity. Note, that the concentration $\tilde{c}$ has no dimension as it is given as part per volume. The parameter $a$ may be called a non-dimensional depth of quench into the two phase region.

If one introduces the non-dimensional variables $c=\tilde{c} / \Gamma, t=\tilde{t} / \tau$ and $x=\tilde{x} / \ell$ with scales

$$
\Gamma=a^{1 / 2}, \quad \tau=\frac{\sigma}{M a^{2} \kappa^{2}} \quad \text { and } \quad \ell=\sqrt{\frac{\sigma}{a \kappa}}
$$

for concentration, time and length, respectively, one obtains the dimensionless Eq. (3). In this way the model parameters are reduced to four: the non-dimensional chemical potential difference

$$
\mu=\frac{\tilde{\mu}}{a^{3 / 2} \kappa},
$$

the non-dimensional plate velocity

$$
V=\frac{\tau}{\ell} \tilde{V}=\frac{\sigma^{1 / 2}}{M a^{3 / 2} \kappa^{3 / 2}} \tilde{V},
$$

the non-dimensional domain size $L=\tilde{L} / \ell$ and the scaled concentration at the meniscus-side boundary $c_{0}$. As the present study aims at understanding the onset of the deposition of line patterns observed in Ref. [22], the same scaling is used although other scalings exist that allow for distinct parametric studies within the same physical parameter space (see note [36]).

The main focus here is the dependence of the solution behaviour on the non-dimensional plate velocity that represents the primary control parameter. If ones main interest is in the influence of temperature (quench depth), as investigated in [19], the scaling sketched in the note [36] is more adequate. Here, the temperature dependent parameter $a$ appears in all scales and dimensionless numbers (7) - (9).

The resulting bifurcation diagram in terms of our primary control parameter shall be complemented by a study of transitions triggered by changing a second parameter that represents a distance to the threshold of structure formation at equilibrium. In the given scaling, we chose the dimensionless domain size $L$, i.e., the ratio of the physical domain size $\tilde{L}$ to the typical patterning length $\ell$. If one assumes the 'undercooling' $a$ and energy scale $\kappa$ are fixed, changing $L$ amounts to a change of the interface tension $\sigma$. We note, however, that it is difficult in any experimental 
realisation to change $\sigma$ without changing $\kappa$ and $a$ as well. Although, one could chose $\mu$ or $c_{0}$ as second parameter, we think that this does not allow for such a clear physical interpretation as does the choice of $V$ and $L$ as control parameters. The two-parameter study in $V$ and $L$ presented in section III elucidates how the intertwined structure of snaking steady states and time-periodic solutions emerges.

\section{Numerical approach}

For the numerical continuation [37-39] of steady and time-periodic solutions of Eq. (3) with BC (5), we employ spatial discretization onto an equidistant grid and approximate the PDE by a large number of ODEs, one for each grid point. Here, we choose second order finite differences to approximate spatial derivatives, that is, exactly the same discretization scheme that has been used in the direct numerical simulations in Ref. [22]. Due to the simplicity of this scheme, the boundary conditions of the problem can be easily implemented what would, for example, not be the case with spectral Chebychev methods.

As a result we obtain a dynamical system consisting of $n$ ODEs. It describes the time evolution of the concentration values $c_{i}$ at equidistant points $x_{i}$. We use the package auto07p [37, 40] to (i) perform pseudo-arclength continuation [41] of fixed points of the dynamical system that correspond to steady solution profiles $c_{0}(x)$ of the PDE; to (ii) detect saddle-node and Hopf bifurcations of the fixed points, and most importantly (iii) to continue stable and unstable time-periodic solutions of the dynamical system that correspond to time-periodic solutions of the PDE that represent the deposition of regular line patterns. Furthermore we track (iv) the loci of saddle-node and Hopf bifurcations in an appropriate two-parameter plane.

Note that the unstable time-periodic solutions are not accessible by direct numerical integration since the numerical treatment necessarily involves fluctuations. However, the structure of the bifurcation diagram can only be completely understood if the unstable solutions are included. Continuation has previously been employed for kinetic equations like Eq. (3) and recent examples include studies of depinning droplets in 1d [42] and 2d [43, 44], of drawn menisci of simple liquids [45] and of finite-time sigularities in film rupture [46]. A brief overview is given in section 4.2 of Ref. [39].

The solutions of the model equations are vectors in a high dimensional space. In order to visualize and further analyse the bifurcation behaviour of the system, we define as solution measure 


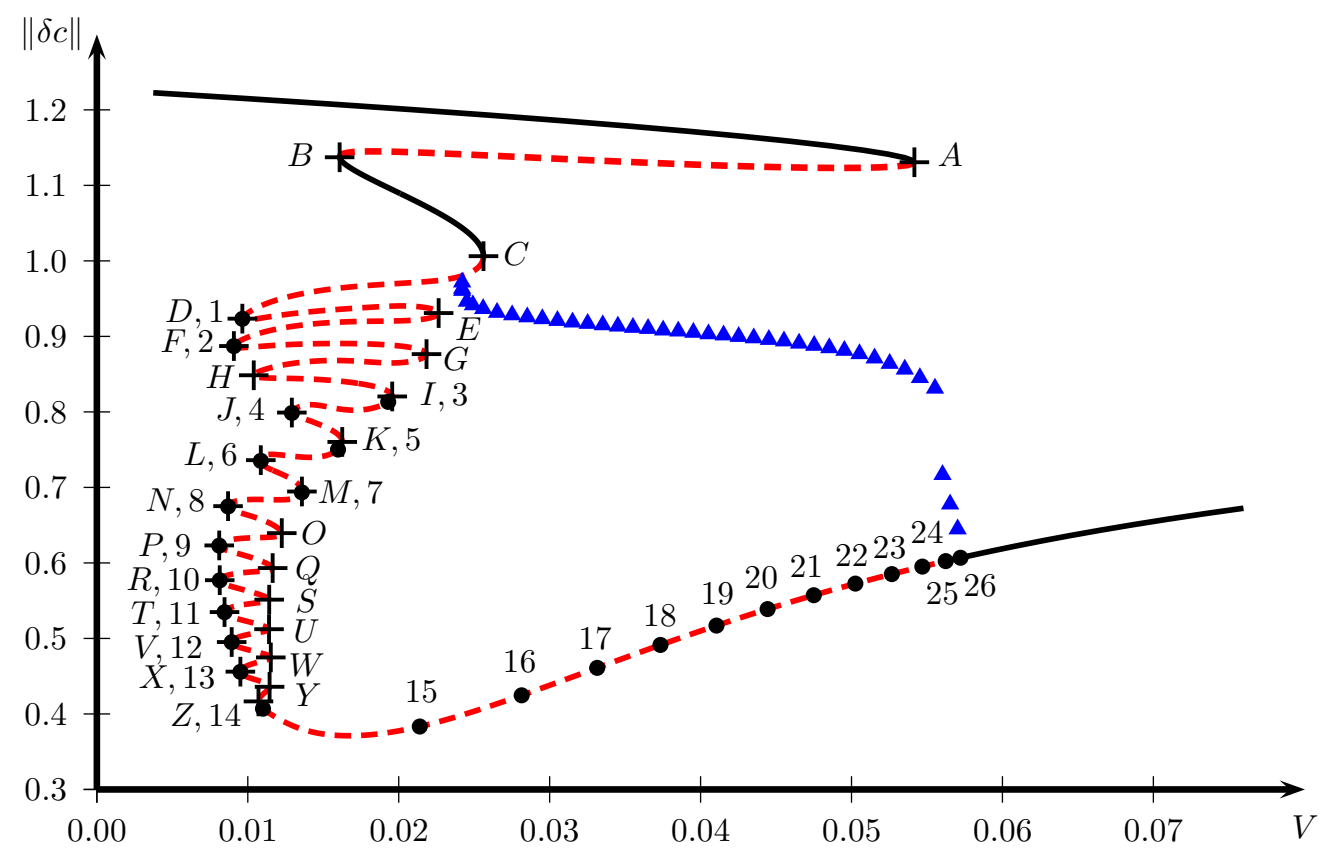

FIG. 2: Shown is the (time-averaged) $\|\delta c\|$ norm of steady and time-periodic solutions of Eq. (3) in dependence of the dimensionless plate velocity $V$ for $L=100, \mu=0.5, x_{s}=10, l_{s}=2$ and $c_{0}=-0.9$. The solid and dashed lines represent stable and unstable steady states (homogeneous deposition), respectively, as obtained by numerical path continuation. The blue triangles correspond to time-periodic solutions (line deposition) obtained by direct numerical simulation. Letters A-Z label saddle-node bifurcations (folds), while numbers 1-26 label Hopf bifurcations (HB). Selected steady profiles are given in Fig. 3.

the norm

$$
\|\delta c\|=\left[\frac{1}{T L} \int_{0}^{T} \int_{0}^{L} c(x, t)^{2} d x d t\right]^{1 / 2}
$$

where $L$ is the domain size and $T$ the temporal period for the time-periodic solutions. For steady states and when investigating system trajectories, the time average $(1 / T) \int d t \ldots$ is omitted.

\section{RESULTS}

\section{A. Steady profiles}

In our previous investigation [22] a combination of direct numerical simulation and numerical continuation of steady solutions was used to construct a preliminary bifurcation diagram with the plate velocity $V$ as primary control parameter, as summarised in Fig. 2. The diagram includes a family consisting of stable and unstable steady states (solid black and dashed red lines, respec- 

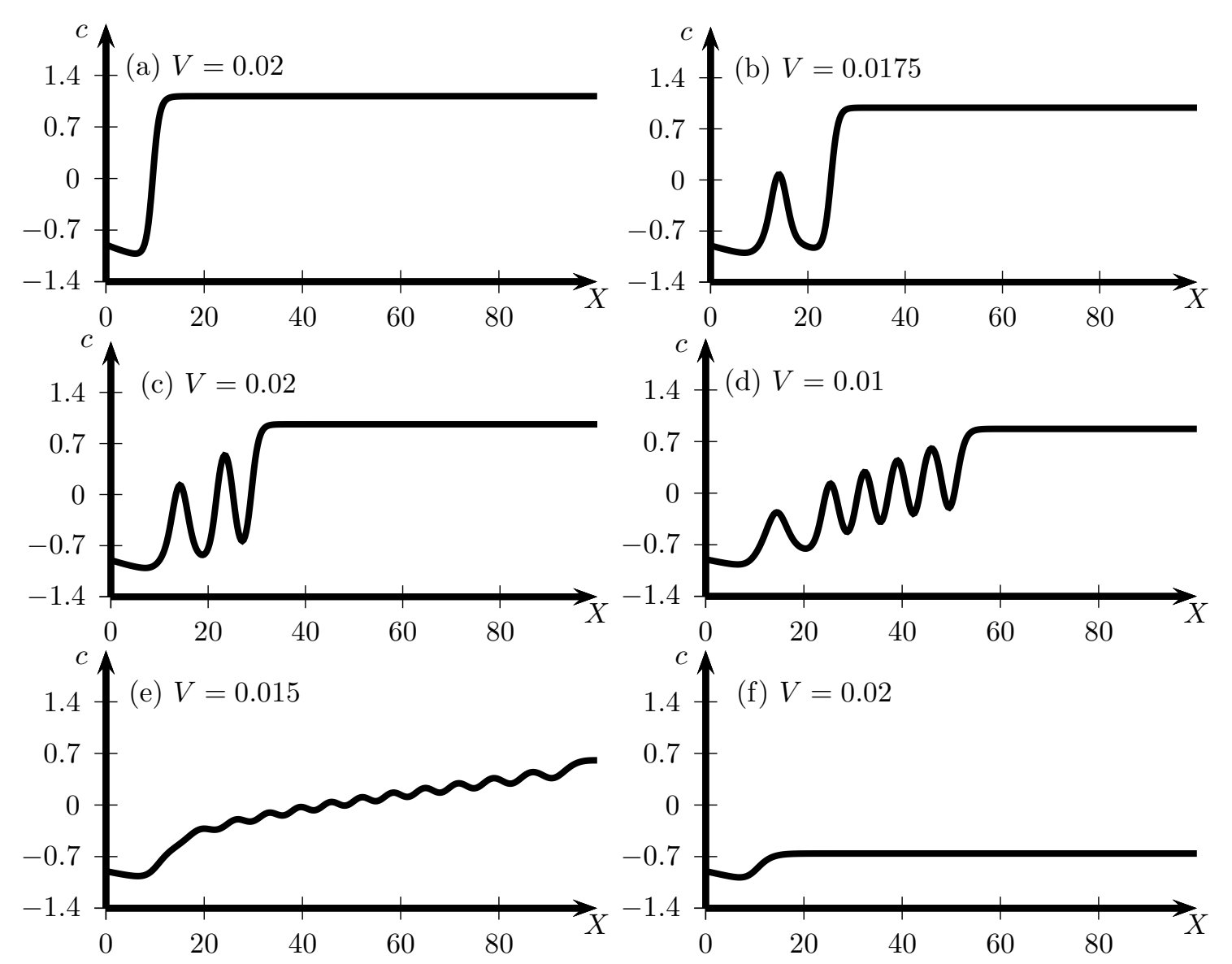

FIG. 3: Examples of steady solution profiles from various points along the family of steady solutions in Fig. 2: (a) between the folds $B$ and $C$, (b) between the folds $D$ and $E$, (c) between the folds $G$ and $H$, (d) between the folds $L$ and $M$, (e) between the folds $Y$ and $Z$, (f) to the right of Hopf bifurcation 26 .

tively), and a branch of stable time-periodic solutions (blue triangles) that seems discontinuous close to its end at large $V$. The parameters employed with Eq. (3) are $L=100, \mu=0.5, x_{s}=10$, $l_{s}=2$ and $c_{0}=-0.9$. With the exception of $L$ these values are kept throughout the present study. A linear stability analysis of the steady solutions provides the loci of 26 Hopf bifurcations (HB) that are numbered 1-26 in Fig. 2. The 26 saddle-node bifurcations (folds) of the snaking curve are labeled by letters $A-Z$. Note that the various saddle-node and Hopf bifurcations are not created by a few eigenvalues that repeatedly cross the imaginary axis. Instead, nearly all of the Hopf bifurcations that are situated on the steady state branch left of the point of minimal norm (at $\left.V_{\min } \approx 0.015\right)$ in Fig. 2 are related to different pairs of complex conjugate eigenvalues that aquire a positive real part. All of them become stable again on the piece of steady state branch right of $V_{\min }$, giving rise to more Hopf bifurcations. The steady profile is linearly stable for $V \gtrsim 0.06$ 
where the last HB occurs. Therefore our further study focuses on the velocity range $0<V<0.1$ where all the mentioned bifurcations occur. Selected steady states profiles are shown in Fig. 3.).

On the snaking structure one observes two basic scenarios for the relation of saddle-node and Hopf bifurcations: In scenario (i) first a negative real eigenvalue crosses zero at a saddle-node bifurcation rendering the corresponding eigenmode unstable, then the same eigenvalue becomes negative again at a second saddle-node bifurcation, briefly before forming a complex conjugate pair together with another negative real eigenvalue. The complex conjugate pair then crosses the imaginary axis at a Hopf bifurcation. Examples for this scenario are all such sequences between from $(O, P, 9)$ to $(Y, Z, 14)$ in Fig. 2. As the second saddle-node and the Hopf bifurcation are often very close to each other the system might be very close to a codimension 2 BogdanovTakens bifurcation. In scenario (ii) first a complex conjugate pair of eigenvalues with negative real part crosses the imaginary axis at a Hopf bifurcation. The pair then splits into two positive real eigenvalues. The smaller of the two becomes negative at a saddle-node bifurcation before it joins another negative real eigenvalue to become another complex conjugate pair of eigenvalues. Briefly afterwards the pair crosses the imaginary axis at a second Hopf bifurcation. This second Hopf bifurcation can form the first Hopf bifurcation of the next such sequence. Examples for this scenario are all such sequences from $(3, J, 4)$ to $(7, N, 8)$ in Fig. 2.

Next, in section III B, we complete the bifurcation diagram for $L=100$, by a systematic continuation of all branches of time-periodic solutions that emerge at the described Hopf bifurcations. Then, in section IIIC, we turn our attention to the snaking structure in the range $0.005 \lesssim V \lesssim 0.025$ of the bifurcation diagram. To better understand the relation of the saddlenode and Hopf bifurcations of the branch of steady solutions, we investigate how these structures and the time-periodic branches emerge/vanish when changing the dimensionless domain size $L$.

\section{B. Completed bifurcation diagram for $L=100$}

To complete the bifurcation diagram Fig. 2, i.e., the case of $L=100$ that is also investigated in Ref. [22], we continue all the branches of time-periodic solutions emerging at the Hopf bifurcations that we call 'primary Hopf branches', as well as selected branches of time-periodic solutions emerging from the primary Hopf branches in period-doubling bifurcations. The latter we call 'secondary Hopf branches'. As a result we find the harp-like structure presented in Fig. 4. Each primary Hopf branch that starts either sub- or supercritically at HB 15 to HB 26, i.e., right 


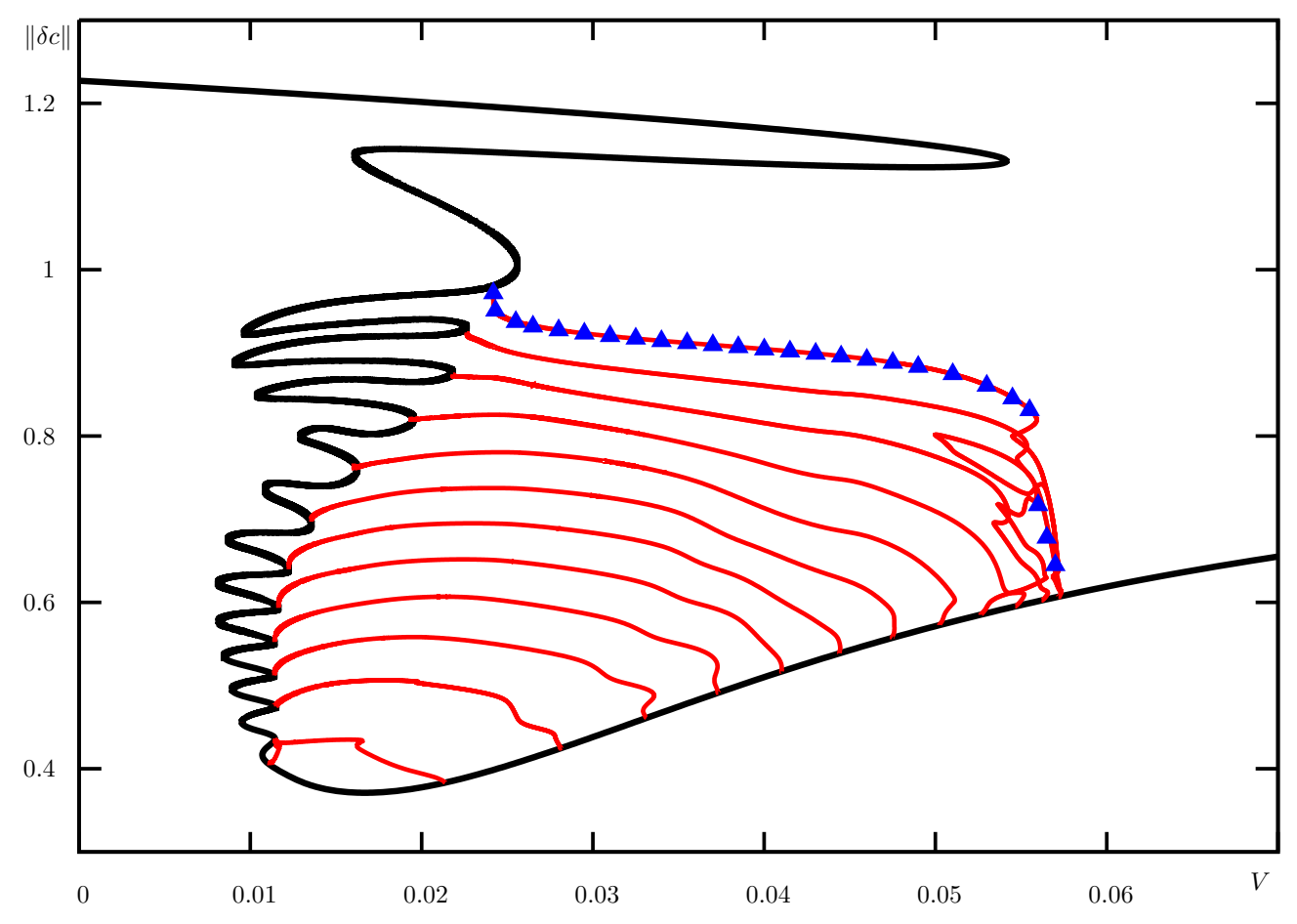

FIG. 4: Shown is the (time-averaged) $\mid \delta c \|$ norm of steady and time-periodic solutions of Eq. (3) in dependence of the dimensionless plate velocity $V$ for $L=100$ and all the remaining parameters as in Fig. 2 . The thick black solid and thin red solid lines represent steady states (homogeneous deposition) and timeperiodic solutions (line deposition), respectively, as obtained by numerical path continuation. The blue triangles correspond to time-periodic solutions obtained by direct numerical simulation. Selected zooms showing important details are given in Figs. 5 and 6.

of the point where the norm is minimal, eventually turns towards smaller $V$, continues to lower $V$ ('spanning the harp') and finally ends in a global bifurcation on the snaking part of the branch of steady states. The global bifurcation is either a saddle-node infinite period (sniper) bifurcation or a homoclinic bifurcation [38, 47]. There are several secondary Hopf branches that connect a period doubling bifurcation of a primary Hopf branch (e.g., solid red and dashed blue branches in Fig. 6) either to a global bifurcation on the snake (e.g., branch that connects the first PD of the HB 26 branch to a homoclinic bifurcation near fold $C$ ), or to a primary $\mathrm{HB}$ (e.g., branch that connects the second PD of HB 26 to HB 24, see Fig. 6 (a)) or to another period doubling bifurcation of a primary Hopf branch (see Fig. 10 (b) below). Beside these extended branches, there are (very) short branches that emerge at HB 1 to HB 13 close to folds $D, F, I-N, P, R, T, V, X$ and $Z$ and connect to a global bifurcation nearby (beyond the next fold of the snaking structure). The following list 

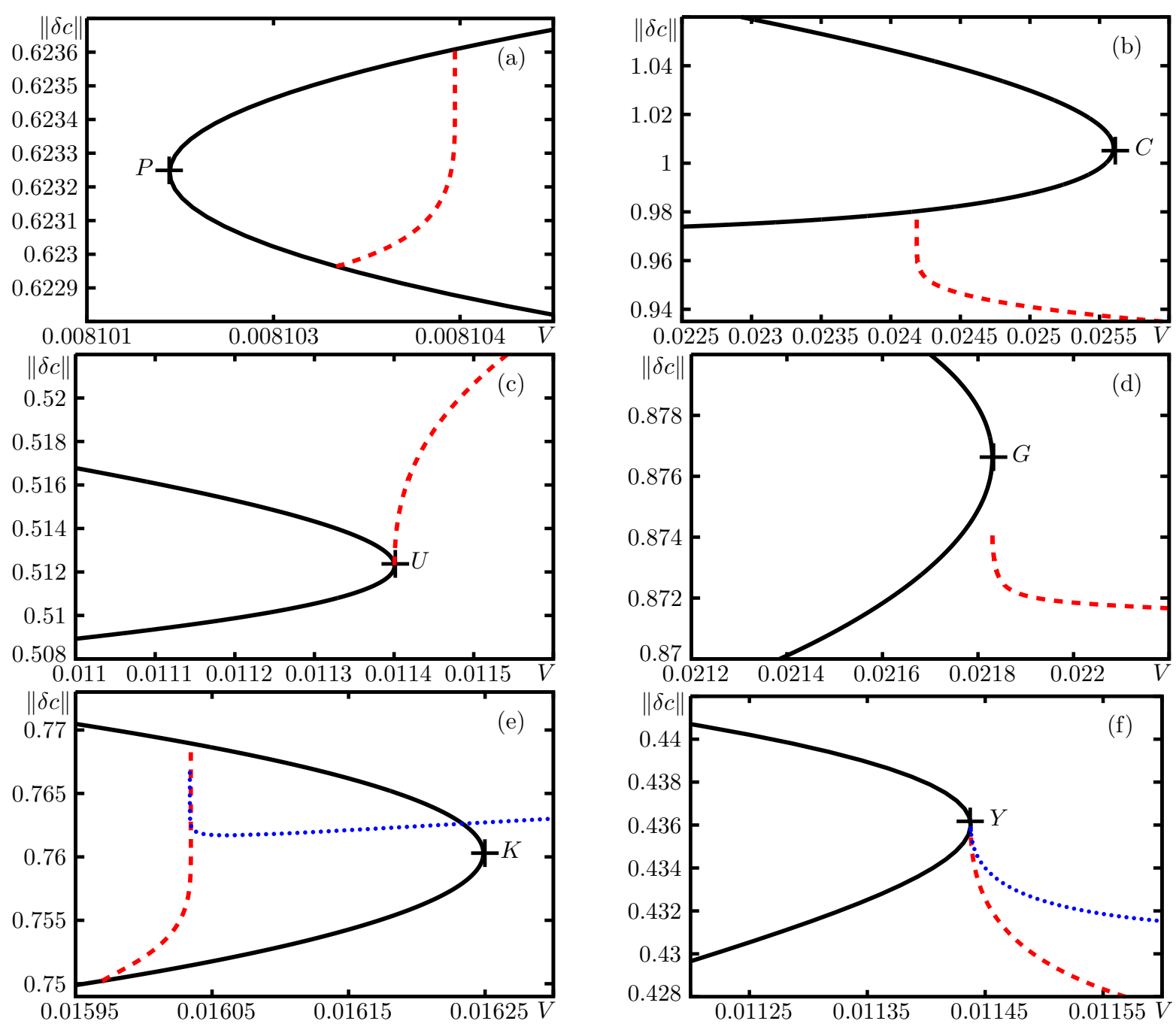

FIG. 5: Close-ups of the bifurcation diagram Fig. 4 zooming in on qualitatively different endpoints of branches of time-periodic solutions that involve global bifurcations. Panels (a) to (f) correspond to cases (i1), (i2), (ii1), (ii2), (iii) and (iv) discussed in the main text.

summarises the features of the five different scenarios in which global bifurcations are observed at low $V$ for branches that emerge from Hopf bifurcations or period-doubling bifurcations at higher $V$.

(i) The branch of time-periodic solutions monotonically approaches a vertical asymptote at some critical $V$ and ends in a homoclinic bifurcation at an unstable steady state on the snake. One may distinguish sub-cases (i1) and (i2) with the branch of time-periodic states approaching the homoclinic bifurcation from the left and from the right, respectively. The case (i1) are the short branches emerging from HB 1, 2, 4, 6 and 8-13 that lie completely 
within one bend of the snake. For an example see Fig. 5 (a) (note the tiny $V$ range). The case (i2) are the short branches emerging from HB 3, 5 and 7 and the long branch that emerges from a period doubling bifurcation of the HB 1 branch (see Fig. 5 (b)). The latter branch consists mainly of the stable time-periodic solutions observed by direct time simulations [22].

(ii) The branch of time-periodic solutions monotonically approaches a vertical asymptote at a critical $V$ that corresponds to a saddle-node bifurcation of the snake of steady states and ends in a sniper bifurcation. One may distinguish sub-cases (ii1) and (ii2) with the branch of time-periodic states approaching the saddle-node bifurcation from above in the $V-\|\delta c\|$ plane and from below, respectively. The case (ii1) are the branches emerging from HB 1621. For an example see Fig. 5 (c). The case (ii2) are the branches coming from HB 15, 25, and 26 (see Fig. 5 (d)).

(iii) Two branches of time-periodic solutions monotonically approach seemingly the same vertical asymptote at a critical $V$ where they end in two homoclinic bifurcations. This occurs for the branches that emerge from HB 3 and 24 and end at the branch between folds $H$ and $I$ as well as for the branches that emerge from HB 5 and 22 and end at the branch between folds $J$ and $K$ (see Fig. 5 (e). For our calculations the respective two homoclinic bifurcations occur at values of $V$ that are identical up to 4 significant digits.

(iv) As (iii), but the two branches end in a sniper bifurcation at a fold (or in a sniper bifurcation and a homoclinic one very close nearby. This occurs for the branches that emerge from HB 14 and 15 (see Fig. 5 (f)). For our calculations the two bifurcations occur at values of $V$ that are identical up to 5 significant digits. As we will explain later, this scenario is related to a codimension 2 bifurcation that creates the cases (i) and (ii).

The information regarding the branches of time-periodic solutions contained in Figs. 4 to 6 goes well beyond Ref. [22] and sheds a new light on results obtained there. In particular, a weakly nonlinear analysis of the branch emerging at the Hopf bifurcation at the highest velocity (HB 26) led to the conclusion, that this HB is subcritical. However, in Ref. [22] it was also observed through time simulations, that the phase space trajectories of the system close to HB 26, where only one pair of complex conjugate modes becomes unstable, are very complicated: First, they exhibit a long phase of slow exponential growth (outwards spiral on a plane in phase space). After 

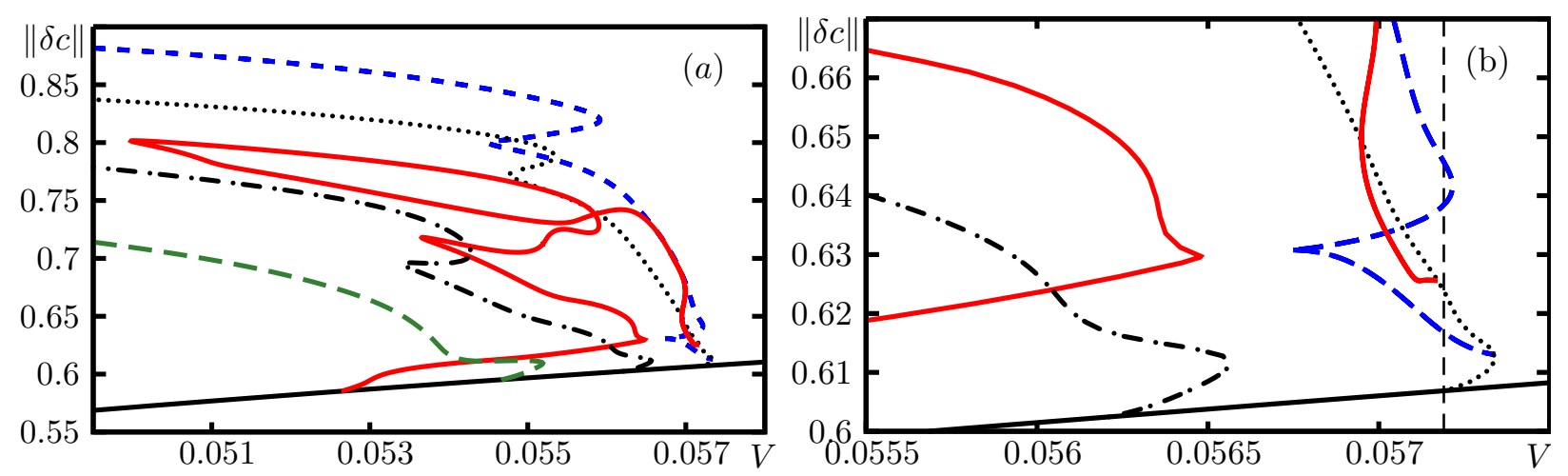

FIG. 6: Close-ups of the bifurcation diagram Fig. 4 zooming in on period doubling bifurcations and complex reconnections in the region $0.55<V<0.58$. Shown are all branches of periodic solutions that emerge from Hopf bifurcation (HB) 26 (dotted), HB 25 (dot-dashed), HB 24 (green long dashed), HB 23 (red solid line), as well as a secondary branch (blue short dashed) emerging from the first period doubling bifurcation of the HB 26 branch. The stable time-periodic solutions observed in the direct time simulations lie on this secondary branch. Note that all the Hopf bifurcations at high velocities are subcritical. The vertical dashed line in panel (b) marks the velocity $V=0.5719$ that is used for the time simulation shown in Fig. 7 and indicates the coexisting solutions at this velocity.

this initial phase, deviations from this planar dynamics are observed in the form of large excursions from the spiral which are then caught by a higher dimensional structure on which the trajectory eventually returns to the plane and begins to follow the spiral, again. This dynamics is not simply periodic and might be chaotic. An attempt to understand this behaviour as an example of a scenario described by Ovsyannikov and Shilnikov [48], which involves a stable and an unstable pairs of complex conjugate eigenvalues failed because it was found that there is a strong interaction of the unstable mode and more than three of the stable modes [22] .

In view of the close-ups of Fig. 4 in Fig. 6, the complicated trajectories and the higher dimensional structure which attracts the dynamics can be understood as a consequence of the existence of several (up to 12) stable and unstable time-periodic solutions at the same velocity value. The majority of these solutions is unstable. As the system spirals away from the unstable fixed point (steady state solution) it feels the attracting and repelling presence of the various coexisting timeperiodic solutions nearby. This can actually be seen in the results of direct numerical simulations performed at a value of $V$ close to HB 26: Once the initial amplification has become large enough, the system behaviour shows clear similarities to the time-periodic branches. This is indicated in 


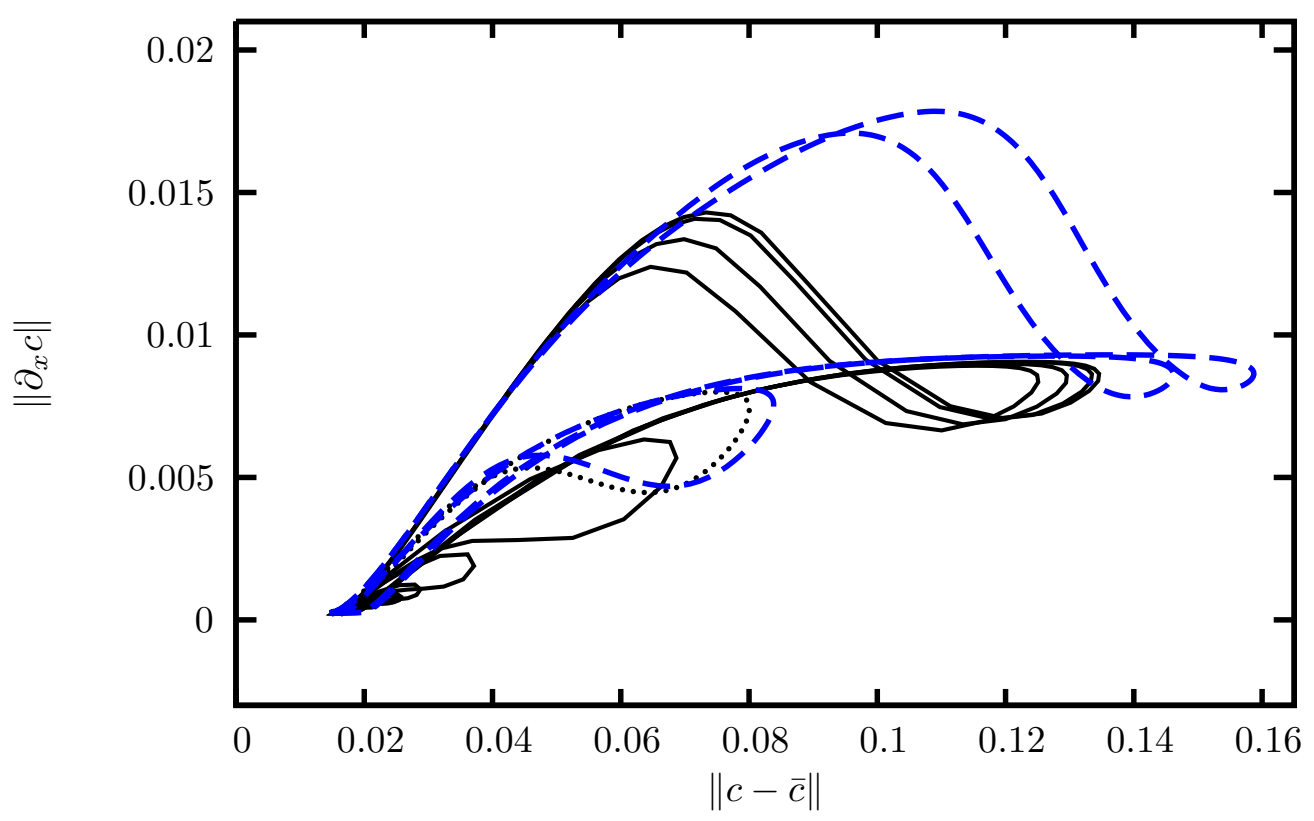

FIG. 7: Shown are representations of various time-dependent solutions on the plane spanned by the norms of $c(x, t)-\bar{c}$ and of $\partial_{x} c(x, t)$, where $\bar{c}$ is the mean value of $c$. A trajectory obtained by direct numerical simulation is given as a thin solid line while the time-periodic branches obtained by numerical continuation are shown in the same linestyles used in Fig. 6. The value of the plate velocity is $V=0.05719$.

Fig. 7 that shows phase plane representations of a simulated trajectory and of the various coexisting time-periodic branches at $V=0.05719$. One notes that although the trace of the simulated time evolution is similar to the time-periodic solutions, it does not approach any of them closely, indicating that they act as repellers. Even in very long simulations, the trajectory does not settle onto any of the stable time-periodic solutions, but eventually returns to a phase space region close to the unstable fixed point where the exponential amplification sets in again. Note that the role of a large number of repellers in the transition to chaos has been analysed, e.g., in Ref. [49] for channel flow.

In the present section we have analysed the bifurcation structure for fixed $L=100$ employing $V$ as main control parameter. To further understand how the observed harp-like structure emerges we next investigate how the bifurcation structure changes with our second control parameter $L$. 

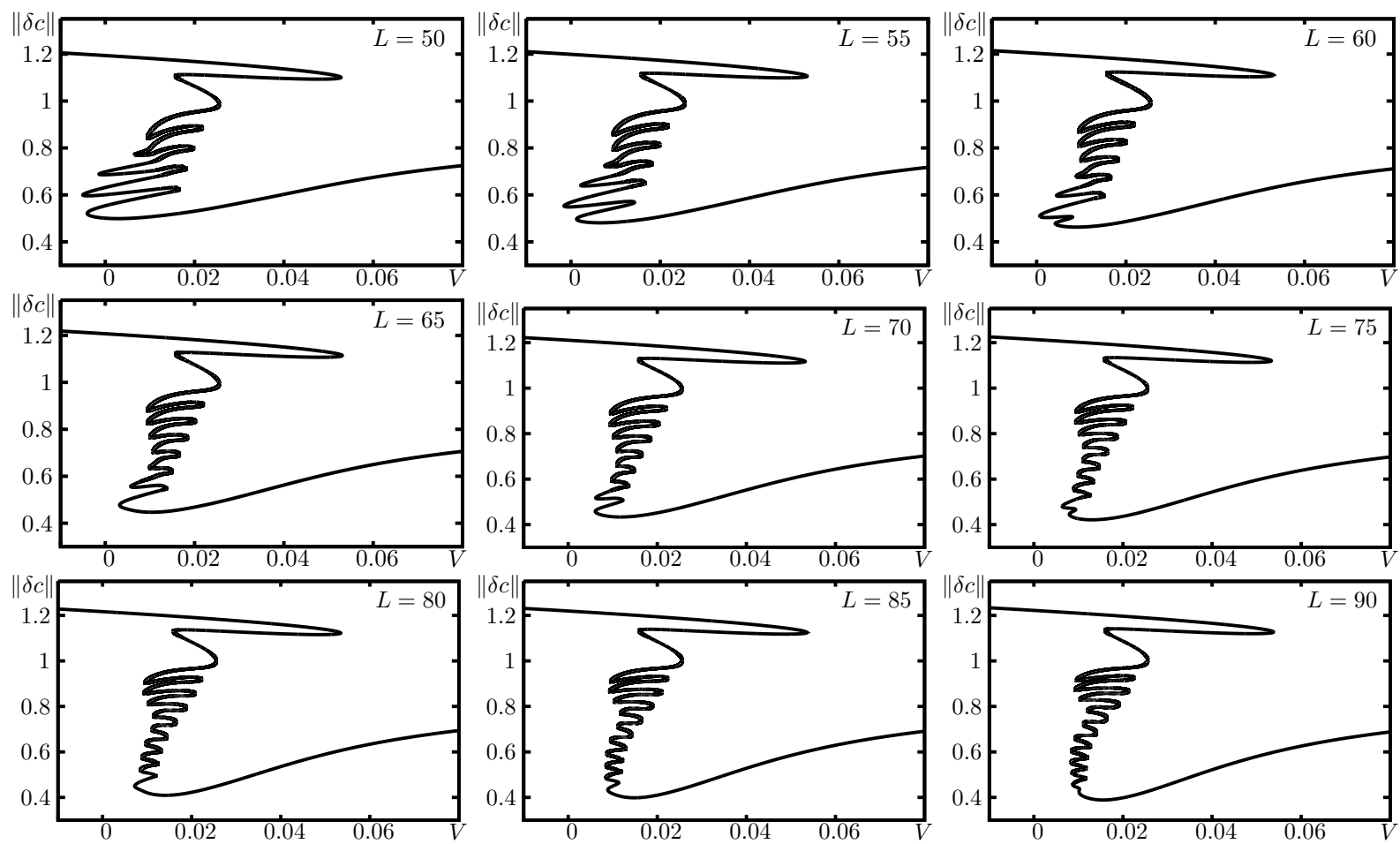

FIG. 8: Shown is the branch of steady solutions for equally spaced values of the domain size from $L=50$ to $L=90$ as indicated in the individual panels (a) to (i). All the remaining parameters are as in Fig. 2. The occurring simplification with decreasing $L$ is discussed in the main text. Fig. 10 show close-ups of the final bends of the snaking structure including the time-periodic branches for $L=90$ to $L=100$.

\section{Simplification of bifurcation structure with decreasing domain size $L$}

Up to here we have completed the bifurcation diagram Fig. 2, i.e., in the case of $L=100$ partially given in Ref. [22], by a systematic continuation of the branches of time-periodic solutions emerging at all of the $26 \mathrm{HB}$. This has allowed for a classification of possible endings of branches of time-periodic solutions corresponding to the behaviour at the onset of line deposition. Overall, the analysis indicates that our particular system has a complicated bifurcation structure that involves saddle-node and Hopf bifurcations of steady states, period doubling bifurcations of timeperiodic solutions and various global bifurcations, namely sniper and homoclinic bifurcations.

These observations are in line with observations in the literature for a wider class of related systems that show deposition or depinning phenomena. For other line deposition phenomena, similar bifurcations are hypothesised based on time simulations in Refs. [13, 14]. In particular, Ref. [14] discusses in which way such line deposition patterns may be seen as resulting from depinning 
transitions, thereby relating the phenomena to the depinning of driven droplets on heterogeneous substrates [42, 44,50,51], rotating cylinders [52], or to the depinning of interface undulations from the advancing tip of an air finger in a liquid-filled channel [53, 54]. In these related systems depinning can also occur through Hopf, sniper or homoclinic bifurcations. This will be further discussed in the conclusion.

The main physical ingredients of our particular system are a first order phase transition and a lateral driving (towards the right hand side, see Fig. 1) that keeps the system permanently out of equilibrium. Close to the left boundary (the meniscus side), the underlying energy is a symmetric double well. As the concentration on the boundary is fixed to a value close to one of the minima of the energy, the corresponding phase is preferred. This changes to the right of the critical position $x_{\mathrm{s}}$ where the double well potential is tilted such that the other phase is preferred.

Considering our main influences, we already know that in the case without driving $V=0$ one obtains a standard system that evolves towards equilibrium, i.e., in the right hand part it approaches the high-concentration phase promoted by the tilted double well potential. Out of equilibrium one finds at low [large] $V$ a homogeneous deposition of the preferred phase [the boundary phase]. At intermediate $V$ the interplay of driving and phase dynamics results in stripe deposition.

One can also expect that no structure formation will occur if the intrinsic length scale of the patterning $\ell$ is larger than the considered domain size $\tilde{L}$. This implies that the rich structure of the bifurcation diagram Fig. 4 should vanish step by step if $L=\tilde{L} / \ell$ is decreased. In the following, we focus on this stepwise simplification and, in order to observe it, we compute bifurcation diagrams for decreasing dimensionless domain size $L$ that represents the ratio of dimensional domain size and intrinsic patterning length scale. In the alternative scaling discussed in note [36], one would keep $L$ fixed and reduce the quench depth $a$.

We focus on the range $50 \leq L \leq 100$ as for smaller $L$ the distance $x_{s}$ between the boundary and the position where the tilt of the potential part of the free energy changes becomes important and renders the emerging picture non-generic. Figure 8 shows the branch of steady solutions for various $L$ in the considered range. One notices that with increasing $L$, the snaking structure becomes larger, i.e., more and more pairs of saddle-node bifurcations emerge in subsequent hysteresis bifurcations, adding wiggles to the branch that then widen with increasing $L$. In this way, one passes from 12 folds at $L=50$ to 26 folds at $L=100$. This is of course expected, since with each turn of the snake, a bump is added to the solution profile until the part of the snake with the lowest norm corresponds to a state in which the complete domain to the right of the substrate-mediated 


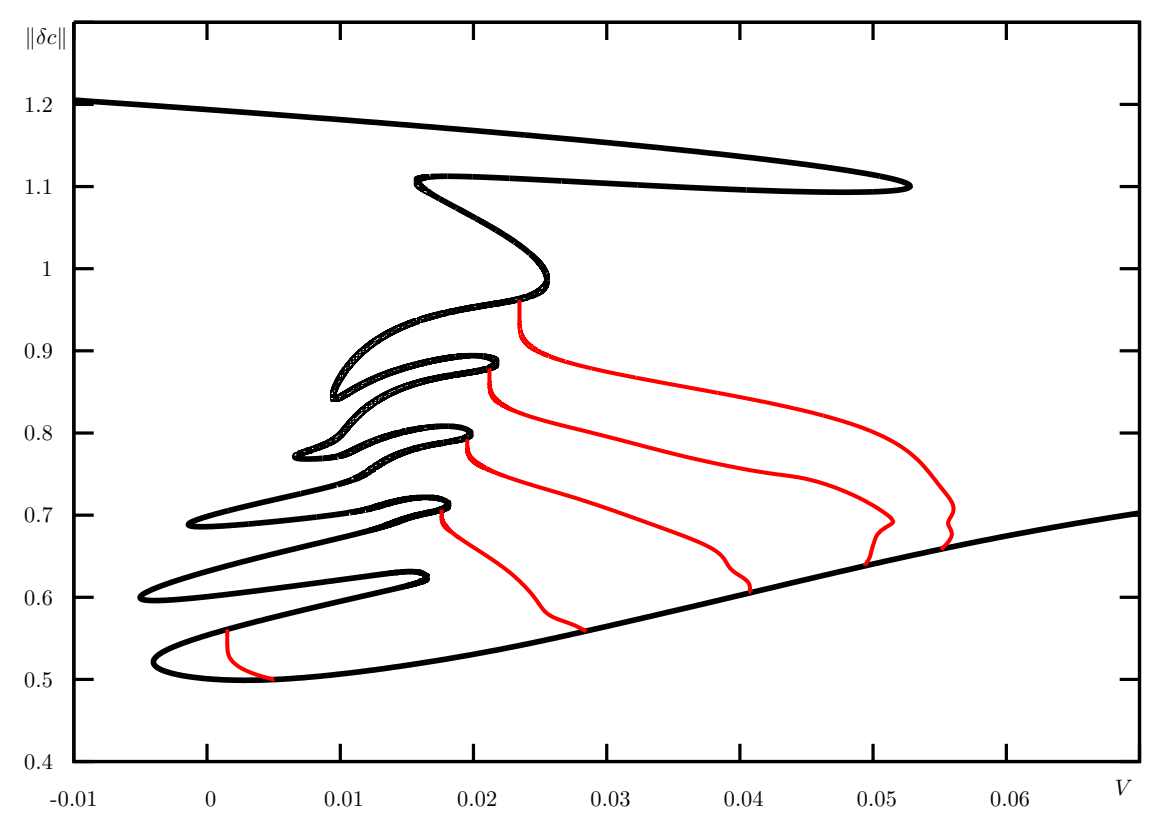

FIG. 9: Shown is the (time-averaged) $\|\delta c\|$ norm of steady and time-periodic solutions of Eq. (3) in dependence of the dimensionless plate velocity $V$ for $L=50$ and all the remaining parameters as in Fig. 2. The thick black solid and thin red solid lines represent steady states (homogeneous deposition) and timeperiodic solutions (line deposition), respectively, as obtained by numerical path continuation. Note, that the reconnection of the rightmost branches that is observed in the case $L=100$ (see Fig. 4) is not observed at $L=50$.

condensation threshold is filled with bumps (see Fig. 3 (e)). With increasing $L$, more bumps fit into the domain, thus prolonging the snake.

However, from the continuation results shown in Fig. 4 one knows that the snaking structure is closely linked to the branches of time-periodic solutions. This raises the question what happens to the branches observed at $L=100$ when the snaking structure shrinks with decreasing domain size $L$. Figure 9 shows the bifurcation diagram with steady and time-peridic solution for $L=50$. One can see a smaller version of the harp-like structure that we observed for $L=100$ in Fig. 4. With decreasing $L$, as more and more wiggles disappear from the branch of steady solutions, more and more branches of time periodic solutions vanish as well and the structure shown in Fig. 4 evolves to the structure shown in Fig. 9.

We observe a generic sequence of transitions that eliminate branches of time-periodic solutions and the related global and local bifurcations in parallel to the elimination of folds of the snake when $L$ is decreased. We explain the mechanism using the example of the time-periodic branches 
of lowest norm in Fig. 4, in particular the ones emerging from HB 13-16. All the involved solution branches are shown for eight values of $L$ between 92 and 100 in Fig. 10 while Fig. 11 shows the loci of the involved Hopf, homoclinic, sniper and saddle-node bifurcations in the parameter plane spanned by $V$ and $L$.

We describe the transitions with increasing $L$, i.e., the various bifurcations and branches are created subsequently. At $L=90$ none of the Hopf bifurcations 13-16 and none of the related time-periodic branches exist, the lowest pair of saddle-node bifurcations of steady states has just been created in a hysteresis bifurcation at $L \approx 89.5$ (lower black solid line in Fig. 11). Slightly below $L=91$ one observes a codimension 2 bifurcation that occurs close to the minimum of the curve of steady states. As a result a pair of Hopf bifurcations (HB 13 and 16) appears together with the branch of time-periodic solutions that connects them. The creation of two HB corresponds to the minimum of the lower red dashed curve in Fig. 11 that indicates the loci of the two HB. With increasing $L$, the two HB move apart and the left (lower $V$ ) HB moves towards the lowest fold of the snake; see the bifurcation diagram at $L=92$ in Fig. 10 (h). At about $L=92.5$, the branch of time-periodic solutions that connects HB 13 and 16 breaks up into two disconnected branches. This happens when the branch of time-periodic solutions develops a protrusion that eventually collides with the unstable steady state slightly beside or directly at the lowest right fold $(W)$. This codimension 2 bifurcation is marked by the lower blue filled circle in Fig. 11. The bifurcation diagram in Fig. $10(\mathrm{~g})$ is at a slightly larger $L$, the two global bifurcations are already separated. The new left branch of time-periodic solutions coming from HB 13 at lower $V$ now ends in a homoclinic bifurcation that moves towards smaller $V$ (dotted line in Fig. 11) while the right branch of time-periodic solutions, coming from HB 16 at larger $V$, terminates in a sniper bifurcation at fold $W$. Further increasing $L$, the left branch becomes shorter and shorter, and practically disappears into fold $X$, while the right branch becomes longer as HB 16 moves continuously towards larger velocities and the other end remains at fold $W$ (see Figs. 10 (a)-(f) and Fig. 11). In the course of this process, there are also two folds created on the branch of timeperiodic solutions that are again annihilated at about $L=95$. Note that the HB and the homoclinic bifurcation that remain very close to the left fold (on opposite sites) could, in principle annihilate (and be recreated) in Bogdanov-Takens bifurcations [38, see section 8.4. of], but whether this actually happens, e.g., between $L \approx 93$ and $L \approx 97$ we are not able to decide on the basis of the present numerical accuracy.

Then the entire process is repeated: At about $L=97.5$, a pair of saddle-node bifurcations of 

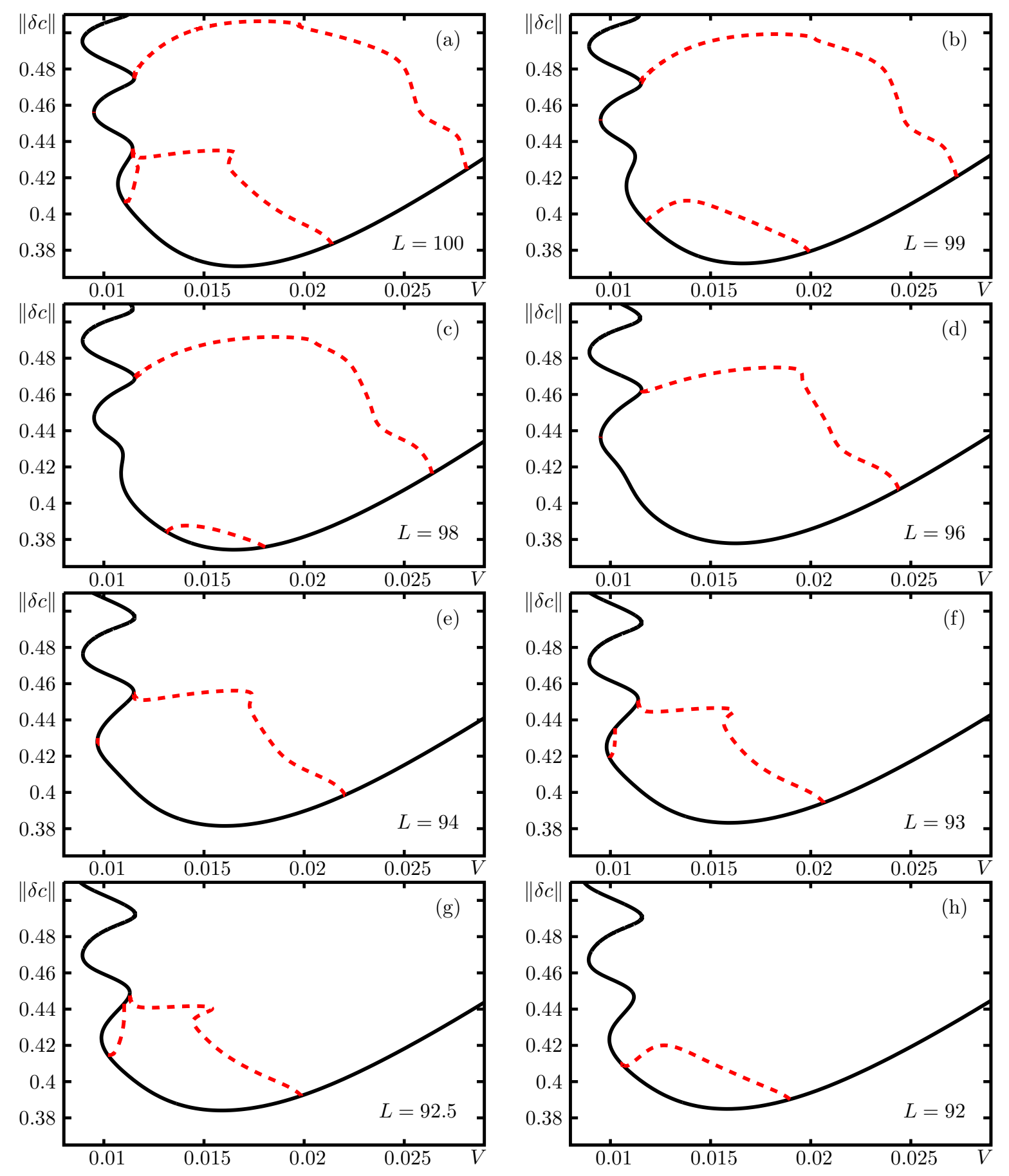

FIG. 10: Shown are close-ups of the lower part of the snaking structure for various values of domain size $L$ from 100 to 90 as indicated in the individual panels (a) to (h). The thick black solid and red dashed lines represent steady states (homogeneous deposition) and time-periodic solutions (line deposition), respectively, as obtained by numerical path continuation. 


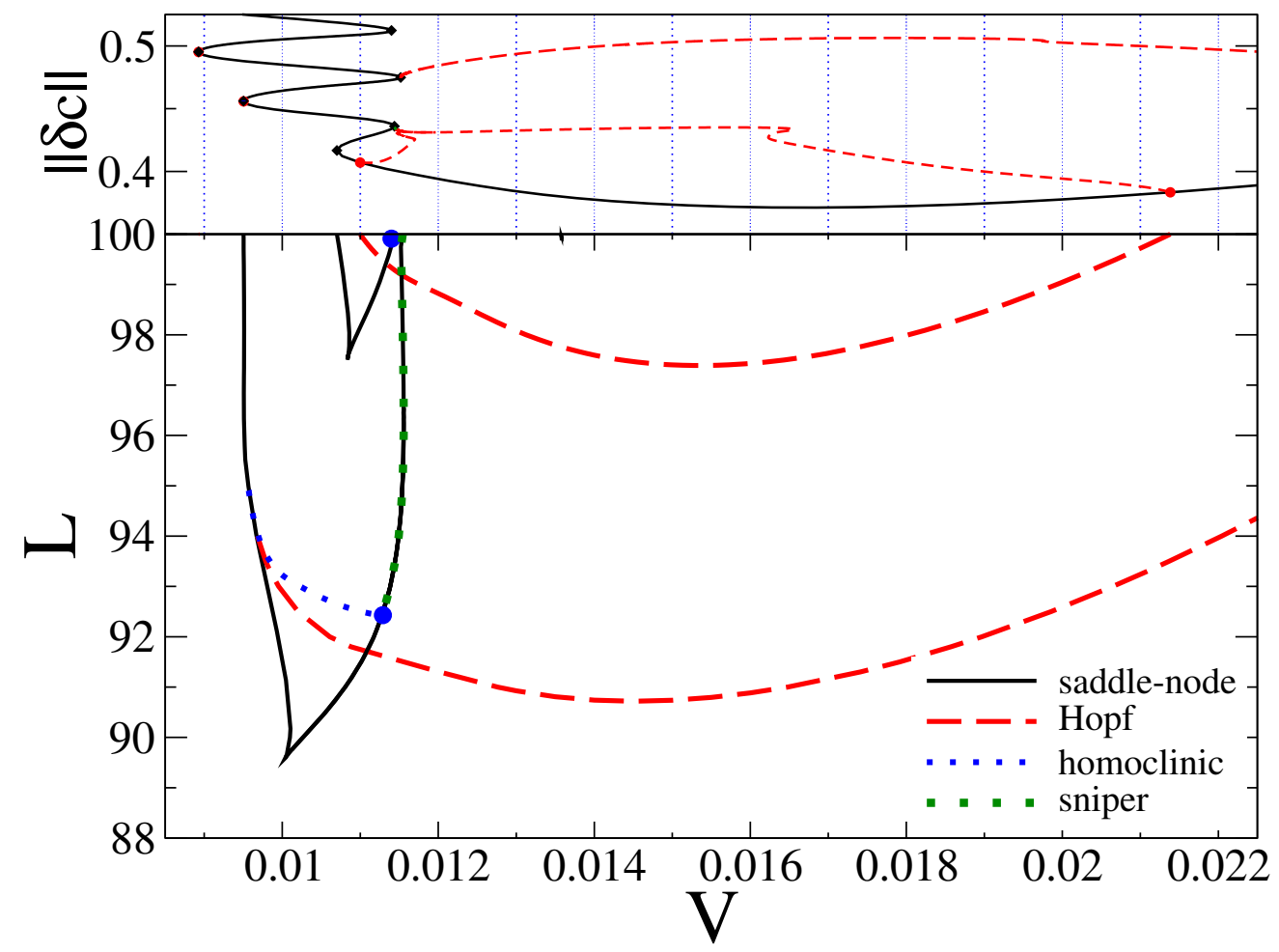

FIG. 11: Loci of the folds $V$ to $Z$ (black solid lines), the Hopf bifurcations 13 to 16 (red dashed lines), and of the homoclinic bifurcation where the primary branch ends that emerges from HB 14 (dotted line). Blue filled circles indicates where a branch of time-periodic solutions collides with the branch of steady states creating a homoclinic and a sniper bifurcation. Above the filled circle a sniper bifurcation coincides with the black solid line of the respective fold.

steady states is created (upper black solid line in Fig. 11) and a pair of Hopf bifurcations (HB 14 and 15) appears together with the branch of time-periodic solutions that connects them (minimum of the upper red dashed line in Fig. 11). The two HB move apart and the left one approaches fold $Z$, and two global bifurcations are created when the branch of time-periodic solutions connects to the steady state branch close to fold $Y$ (see the bifurcation diagrams in Fig. 10 (a)-(c). By this mechanisms, new turns of the snake and new branches of time-periodic solutions can be generated for arbitrary system sizes.

\section{DISCUSSION AND CONCLUSION}

We have explored the bifurcation structure of a modified Cahn-Hilliard equation that describes a system that may undergo a first order phase transition and is kept permanently out of equilibrium 
by a lateral driving. This form of the equation was introduced in Ref. [22] as a model for the deposition of stripe patterns of surfactant molecules through Langmuir-Blodgett transfer, i.e., the transfer of a surfactant monolayer from the free surface of a water bath onto a solid substrate that is drawn out of the bath at a controlled velocity [17]. The pattern formation results from a first order structural liquid-liquid phase transition that is triggered by the intermolecular interactions with the solid plate that become effective when the monolayer gets close to the substrate, i.e., in the contact line region. The effect is called substrate-mediated condensation [16, 17]. The modified Cahn-Hilliard equation represents a reduced model that captures the main features of the patterning as observed before with a hydrodynamic long-wave description in terms of coupled evolution equations for the film height profile and the surfactant concentration [12] that themselves may be seen as a special case of a gradient dynamics description for the coupled evolution of the density of a layer of an insoluble surfactant and of the film thickness profile of the underlying film of liquid [31].

The modified Cahn-Hilliard model captures the main physical ingredients that are the first order phase transition and the lateral driving that together with the pinning boundary condition on the meniscus side keeps the system permanently out of equilibrium. The system is biased towards one of the two phases only beyond a threshold position that represents the position of the contact line region in the physical system. The state in the boundary region on the mensicus side is determined by the boundary condition. In the literature other forms of such Cahn-Hilliard-type models are studied [33-35]. In particular, in these works a front that switches the system between a stable one-phase and an unstable two-phase region of the phase diagram is moved through a fixed domain of given mean concentration. However, the onset of pattern deposition is not studied in a way that could be directly compared to our results. In particular, no bifurcations between steady states and time-periodic states (in the frame moving with the imposed front speed) are discussed.

Our analysis has shown that the snaking of steady states (observed in Ref. [22]) that correspond to various fronts between a homogeneous low concentration region close to the left domain boundary and a homogeneous state far away from the bath is intertwined with a large number of branches of time-periodic solutions that emerge from Hopf bifurcations or period doubling bifurcations and end mainly in global bifurcations (sniper and homoclinic) but sometimes also in other period doubling or Hopf bifurcations. Overall the various branches form a harp-like structure (see Fig. 4).

Beside the particular study of the bifurcation diagram for a fixed value of the domain size we 
have analysed how the bifurcation structure simplifies if one decreases the dimensionless domain size, i.e., increases the typical intrinsic length scale of pattern formation related to the first order transition. We have shown that for decreasing $L$ the rich bifurcation structure vanishes step by step through a number of codimension 2 bifurcations, namely, annihilation of pairs of homoclinic and sniper bifurcations, annihilations of pairs of period doubling bifurcations, and annihilations of pairs of Hopf bifurcations. Beside these transitions involving branches of time-periodic solutions, the front snaking structure of steady solutions reduces by annihilations of pairs of saddle-node bifurcations.

The intriguing question of how the complicated bifurcation scenario at large domain size emerges is not only relevant for the system of Langmuir-Blodgett transfer but for a wider class of systems showing deposition and depinning phenomena. It has been argued in Ref. [14] that the onset of line deposition, i.e., the emergence of branches of time-periodic solutions may be seen as resulting from a depinning transition: When a homogeneous layer is deposited on the moving plate the concentration profile is steady in the laboratory frame and one may say that the concentration profile is pinned. However, when lines are deposited, in the laboratory frame they move away from the bath, i.e., the lines are depinned from the meniscus. This implies that one should not only expect to find similarities between the present study and other studies of the bifurcation structure behind deposition patterns as, e.g., Refs. [13-15] (see also review [3]), but see such similarities as well in studies of driven droplets depinning from substrate heterogeneities $[42,44,50,51]$ and rotating cylinders [52]; studies of driven clusters of interacting colloidal particles depinning in heterogeneous pores [55]; or studies of bubbles advancing in a channel with a central geometrical constraint, where the bubbles develop undulations that are steady in the laboratory frame $[53,54]$. In the frame moving with the uniformly advancing tip of the bubble the undulations depin from the tip similar to the depinning of a deposition pattern from the contact line region. A related effect is also observed in abrasive wawterjet cutting, a technique of cutting material with a focused beam of abrasive particles accelerated by water that is moved across the material $[56,57]$. There is a model based on a Kuramoto-Sivashinsky-type equation that shows a transition from smooth cuts (analogeous to our homogeneous deposition) to ripples and striations patterns (analogeous to our line deposition) $[58,59]$. In this system the undulations represent structures that depin from the moving cutting jet.

In all these systems the transition from steady (pinned) states to time-periodic (depinned) states is also found to occur through sub- or supercritical Hopf, sniper or homoclinic bifur- 
cations. Although transitions between different scenarios where observed, in particular, in Refs. [14, 42, 44, 50], the occurring codimension 2 bifurcations involving time-periodic states have not yet been identified.

For each of the mentioned systems one can find a physically meaningful control parameter whose variation brings the system into a part of the parameter space where no large amplitude structures exist, i.e., the system becomes either steady at all values of the imposed driving (here the plate velocity $V$ ) or only allows for small amplitude 'surface waves' (as in the KuramotoSivashinsky limit of the convective Cahn-Hilliard or thin film equation). However, although depinning and deposition patterns are widely occurring, to our knowledge no systematic study exists for any of the mentioned systems that investigates the emergence/vanishing of the rich bifurcation structure related to depinning or pattern deposition that should characterize these system. Therefore, the results obtained here will be of some relevance to other similar systems as they provide a sound basis for comparison.

The present study is limited in two important ways. On the one hand, it is a study of solutions to a partial differential equation with one spatial dimension only, i.e., the described involved bifurcation structure only captures solutions that are invariant w.r.t. translations along the contact line and is, e.g., not able to capture the transition between the deposition of stripes parallel and perpendicular to the contact line observed in Refs. [12, 22, 23] for Langmuir-Blodgett transfer and in the Cahn-Hilliard-type models where quench fronts are moved through the system [33, 35]. Further large-scale numerical bifurcation studies will be needed to systematically explore the full solution space for out-of-equilibrium amended Cahn-Hilliard-type models allowing for a breaking of the transversal geometry in analogy to such studies for depinning droplets [44, 60]. However, also the latter need to be extended to understand how the entire bifurcation structure vanishes if one goes from partially wetting liquids to completely wetting liquids.

We remark that a future study should also further investigate the influence of the boundary conditions, in particular, on the meniscus side. The need of boundary conditions that allow for a controlled through-flow of material as in the present system is a common complication in dragged plate problems. In the case of simple liquids, recently a central manifold reduction was employed to rigorously derive an asymptotic series for the meniscus side that can then be used to construct boundary conditions that do not introduce an additional domain-size dependence [61]. Such an approach has still to be developed for complex fluids.

On the other hand, the present study is entirely based on numerical path continuation while the 
analytical results obtained in Ref. [22] are entirely based on linear and weakly nonlinear analyses. As it is not to be expected that the uncovered complex bifurcation scenario of the full PDE can be treated analytically, there is a strong need for further simplified models based on ordinary differential equations (ODE) that are able to reproduce the main features described here. We expect that they need to be at least four-dimensional. Note, that the authors of Ref. [44] were able to use selected results of the ODE studies in Refs. [62, 63] to explain certain aspects of the depinning behaviour of droplets, however, also there the 'mapping' remains rather incomplete. We hope that our numerical studies of the bifurcation structure of deposition and depinning processes can motivate the development of generic ODE models for this class of processes.

\section{Acknowledgments}

The authors are grateful to the Newton Institute in Cambridge, UK, for its hospitality during their stay at the programme "Mathematical Modelling and Analysis of Complex Fluids and Active Media in Evolving Domains“" where part of this work was done. MHK acknowledges the support by the Human Frontier Science Program (Grant RGP0052/2009-C). This publication is based in part on work supported by Award No. KUK-C1-013-04, made by the King Abdullah University of Science and Technology (KAUST), and LabEX ENS-ICFP: ANR-10-LABX-0010/ANR-10IDEX-0001-02 PSL*.

[1] R. D. Deegan. Pattern formation in drying drops. Phys. Rev. E, 61:475-485, 2000. doi:10.1103/PhysRevE.61.475.

[2] W. Han and Z.Q. Lin. Learning from "Coffee Rings": Ordered structures enabled by controlled evaporative self-assembly. Angew. Chem. Int. Ed., 51:1534-1546, 2012. doi:10.1002/anie.201104454.

[3] U. Thiele. Patterned deposition at moving contact line. Adv. Colloid Interface Sci., 2013. (online at http://dx.doi.org/10.1016/j.cis.2013.11.002). doi:10.1016/j.cis.2013.11.002.

[4] R. V. Craster and O. K. Matar. Dynamics and stability of thin liquid films. Rev. Mod. Phys., 81:11311198, 2009. doi: 10.1103/RevModPhys.81.1131.

[5] A. Oron, S. H. Davis, and S. G. Bankoff. Long-scale evolution of thin liquid films. Rev. Mod. Phys., 69:931-980, 1997. doi:10.1103/RevModPhys.69.931. 
[6] V. S. Mitlin. Dewetting of solid surface: Analogy with spinodal decomposition. J. Colloid Interface Sci., 156:491-497, 1993. doi:10.1006/jcis.1993.1142.

[7] A. Sharma and R. Khanna. Pattern formation in unstable thin liquid films. Phys. Rev. Lett., 81:34633466, 1998. doi:10.1103/PhysRevLett.81.3463.

[8] U. Thiele. Thin film evolution equations from (evaporating) dewetting liquid layers to epitaxial growth. J. Phys.: Condens. Matter, 22:084019, 2010. doi:10.1088/0953-8984/22/8/084019.

[9] A. Pototsky, M. Bestehorn, D. Merkt, and U. Thiele. Morphology changes in the evolution of liquid two-layer films. J. Chem. Phys., 122:224711, 2005. doi:10.1063/1.1927512.

[10] L. Ó. Náraigh and J. L. Thiffeault. Nonlinear dynamics of phase separation in thin films. Nonlinearity, 23:1559-1583, 2010. doi:10.1088/0951-7715/23/7/003.

[11] U. Thiele, D. V. Todorova, and H. Lopez. Gradient dynamics description for films of mixtures and suspensions: Dewetting triggered by coupled film height and concentration fluctuations. Phys. Rev. Lett., 111:117801, 2013. doi:10.1103/PhysRevLett.111.117801.

[12] M. H. Köpf, S. V. Gurevich, R. Friedrich, and L. Chi. Pattern formation in monolayer transfer systems with substrate-mediated condensation. Langmuir, 26:10444-10447, 2010. doi:10.1021/1a101900z.

[13] L. Frastia, A. J. Archer, and U. Thiele. Dynamical model for the formation of patterned deposits at receding contact lines. Phys. Rev. Lett., 106:077801, 2011. doi:10.1103/PhysRevLett.106.077801.

[14] L. Frastia, A. J. Archer, and U. Thiele. Modelling the formation of structured deposits at receding contact lines of evaporating solutions and suspensions. Soft Matter, 8:11363-11386, 2012. doi:10.1039/C2SM26574E.

[15] F. Doumenc and B. Guerrier. Self-patterning induced by a solutal Marangoni effect in a receding drying meniscus. Europhys. Lett., 103:14001, 2013. doi:10.1209/0295-5075/103/14001.

[16] The first order structural transition is between the liquid-expanded phase and the liquid-condensed phase of the surfactant layer. One speaks of a "substrate-mediated condensation" if for a thick sublayer of water the surfactant layer remains in the liquid-expanded one phase region while on a very thin water layer the presence of the solid substrate underneath lowers the free-energy of the condensed state and thus effectively facilitates condensation [17]. The effect is well studied for the phospholipid DPPC (dipalmitoylphosphatidylcholine), but should in principle occur for any surfactant that exhibits such a first order transition.

[17] H. Riegler and K. Spratte. Structural changes in lipid monolayers during the langmuir-blodgett transfer due to substrate/monolayer interactions. Thin Solid Films, 210-211(Part 1):9-12, 1992. 
doi:10.1016/0040-6090(92)90153-3.

[18] M. Gleiche, L. F. Chi, and H. Fuchs. Nanoscopic channel lattices with controlled anisotropic wetting. Nature, 403:173-175, 2000. doi:10.1038/35003149.

[19] M. H. Köpf, H. Harder, J. Reiche, and S. Santer. Impact of temperature on the LB patterning of DPPC on mica. Langmuir, 27(20):12354-12360, September 2011. doi:10.1021/la202728t.

[20] L. Li, M. H. Köpf, S. V. Gurevich, R. Friedrich, and L. Chi. Structure formation by dynamic selfassembly. Small, 8(4):488-503, 2012. doi:10.1002/smll.201101930.

[21] M. H. Köpf, S. V. Gurevich, and R. Friedrich. Thin film dynamics with surfactant phase transition. Europhys. Lett., 86(6):66003, 2009.

[22] M. H. Köpf, S. V. Gurevich, R. Friedrich, and U. Thiele. Substrate-mediated pattern formation in monolayer transfer: a reduced model. New J. Phys., 14:023016, 2012. doi:10.1088/13672630/14/2/023016.

[23] M. H. Köpf, S. V. Gurevich, and R. Friedrich. Controlled nanochannel lattice formation utilizing prepatterned substrates. Phys. Rev. E, 83(1):016212, Jan 2011. doi:10.1103/PhysRevE.83.016212.

[24] M. Wilczek and S. V. Gurevich. Synchronization in Cahn-Hilliard models for langmuir-blodgett transfer. ArXiv e-prints, 2013. arXiv:1308.6691.

[25] J. Burke and E. Knobloch. Localized states in the generalized swift-hohenberg equation. Phys. Rev. E, 73(5):056211 (15 pages), 2006.

[26] J. Burke and E. Knobloch. Homoclinic snaking: Structure and stability. Chaos, 17:037102, 2007.

[27] E Knobloch. Spatially localized structures in dissipative systems: open problems. Nonlinearity, 21(4):T45-T60, 2008.

[28] U. Thiele, A. J. Archer, M. J. Robbins, H. Gomez, and E. Knobloch. Localized states in the conserved Swift-Hohenberg equation with cubic nonlinearity. Phys. Rev. E, 87:042915, 2013. doi:10.1103/PhysRevE.87.042915.

[29] H. Emmerich, H. Löwen, R. Wittkowski, T. Gruhn, G. I. Tóth, G. Tegze, and L. Gránásy. Phasefield-crystal models for condensed matter dynamics on atomic length and diffusive time scales: an overview. Adv. Physics, 61(6):665-743, 2012. doi:10.1080/00018732.2012.737555.

[30] J. W. Cahn and J. E. Hilliard. Free energy of a nonuniform system. 1. Interfacial free energy. J. Chem. Phys., 28:258-267, 1958. doi:10.1063/1.1744102.

[31] U. Thiele, A. J. Archer, and M. Plapp. Thermodynamically consistent description of the hydrodynamics of free surfaces covered by insoluble surfactants of high concentration. Phys. Fluids, 24:102107, 
2012. doi: $10.1063 / 1.4758476$.

[32] J. W. Cahn. Phase separation by spinodal decomposition in isotropic systems. J. Chem. Phys., 42:9399, 1965.

[33] A. Krekhov. Formation of regular structures in the process of phase separation. Phys. Rev. E, 79:035302, 2009. doi:10.1103/PhysRevE.79.035302.

[34] E. M. Foard and A. J. Wagner. Enslaved phase-separation fronts in one-dimensional binary mixtures. Phys. Rev. E, 79:056710, 2009. doi:10.1103/PhysRevE.79.056710.

[35] E. M. Foard and A. J. Wagner. Survey of morphologies formed in the wake of an enslaved phase-separation front in two dimensions. Phys. Rev. E, 85:011501, 2012. doi:10.1103/PhysRevE.85.011501.

[36] For instance, chosing the scales $\Gamma=(\tilde{\mu} / \kappa)^{1 / 3}, \tau=\sigma / M \kappa^{2 / 3} \tilde{\mu}^{4 / 3}$, and $\ell=\sigma^{1 / 2} / \kappa^{1 / 6} \tilde{\mu}^{1 / 3}$ one obtains Eq. (7) with $M, \sigma, \tilde{\mu}$ and $\kappa$ set to one and without the tildes. The non-dimensional plate velocity is still $V=\tilde{V} \tau / \ell$ and the four remaining nondimensional parameters are $V, a, L$ and $c_{0}$.

[37] E. Doedel, H. B. Keller, and J. P. Kernevez. Numerical analysis and control of bifurcation problems (I) Bifurcation in finite dimensions. Int. J. Bifurcation Chaos, 1:493-520, 1991. doi:10.1142/S0218127491000397.

[38] Y. A. Kuznetsov. Elements of Applied Bifurcation Theory. Springer, New York, 3rd edition, 2010.

[39] H. A. Dijkstra, F. W. Wubs, A. K. Cliffe, E. Doedel, I. F. Dragomirescu, B. Eckhardt, A. Y. Gelfgat, A. Hazel, V. Lucarini, A. G. Salinger, E. T. Phipps, J. Sanchez-Umbria, H. Schuttelaars, L. S. Tuckerman, and U. Thiele. Numerical bifurcation methods and their application to fluid dynamics: Analysis beyond simulation. Commun. Comput. Phys., 15:1-45, 2014. doi:10.4208/cicp.240912.180613a.

[40] E. J. Doedel and B. E. Oldeman. AUTO07p: Continuation and bifurcation software for ordinary differential equations. Concordia University, Montreal, 2009.

[41] H. B. Keller. Numerical solution of bifurcation and nonlinear eigenvalue problems, pages 359-384. Academic Press, New York, 1977.

[42] U. Thiele and E. Knobloch. On the depinning of a driven drop on a heterogeneous substrate. New J. Phys., 8:313, 2006. doi:10.1088/1367-2630/8/12/313.

[43] P. Beltrame and U. Thiele. Time integration and steady-state continuation method for lubrication equations. SIAM J. Appl. Dyn. Syst., 9:484-518, 2010. doi:10.1137/080718619.

[44] P. Beltrame, E. Knobloch, P. Hänggi, and U. Thiele. Rayleigh and depinning instabilities of forced liquid ridges on heterogeneous substrates. Phys. Rev. E, 83:016305, 2011. 
doi:10.1103/PhysRevE.83.016305.

[45] M. Galvagno, D. Tseluiko, H. Lopez, and U. Thiele. Continuous and discontinuous dynamic unbinding transitions in drawn film flow. Phys. Rev. Lett., 2014. (at press). arXiv:1311.6994.

[46] D. Tseluiko, J. Baxter, and U. Thiele. A homotopy continuation approach for analysing finite-time singularities in thin liquid films. IMA J. Appl. Math., 78:762-776, 2013. doi:10.1093/imamat/hxt021.

[47] S. H. Strogatz. Nonlinear Dynamics and Chaos. Addison-Wesley, 1994.

[48] I M Ovsyannikov and L P Shil'nikov. Systems with a homoclinic curve of multidimensional saddlefocus type, and spiral chaos. Mathematics of the USSR-Sbornik, 73(2):415-443, 1992. url: http: //stack.iop.org/0025-5734/73/i=2/a=A07.

[49] B. Eckhardt and A. Mersmann. Transition to turbulence in a shear flow. Phys. Rev. E, 60:509-517, 1999.

[50] D. Herde, U. Thiele, S. Herminghaus, and M. Brinkmann. Driven large contact angle droplets on chemically heterogeneous substrates. Europhys. Lett., 100:16002, 2012. doi:10.1209/02955075/100/16002.

[51] S Varagnolo, D Ferraro, P Fantinel, M Pierno, G Mistura, G Amati, L Biferale, and M Sbragaglia. Stick-slip sliding of water drops on chemically heterogeneous surfaces. Phys. Rev. Lett., 111:066101, 2013. doi:10.1103/PhysRevLett.111.066101.

[52] U. Thiele. On the depinning of a drop of partially wetting liquid on a rotating cylinder. J. Fluid Mech., 671:121-136, 2011. doi:10.1017/S0022112010005483.

[53] M Pailha, AL Hazel, PA Glendinning, and A Juel. Oscillatory bubbles induced by geometrical constraint. Phys. Fluids, 24:021702, 2012. doi:10.1063/1.3682772.

[54] A. B. Thompson, A. Juel, and A. L. Hazel. Multiple finger propagation modes in hele-shaw channels of variable depth. J. Fluid Mech., 2014. at press.

[55] A. Pototsky, A. J. Archer, S. E. Savel'ev, U. Thiele, and F. Marchesoni. Ratcheting of driven attracting colloidal particles: Temporal density oscillations and current multiplicity. Phys. Rev. E, 83:061401, 2011. doi:10.1103/PhysRevE.83.061401.

[56] Mohamed Hashish. Visualization of the abrasive-waterjet cutting process. Experimental Mechanics, 28(2):159-169-, 1988.

[57] Janet Folkes. Waterjet - an innovative tool for manufacturing. Journal of Materials Processing Technology, 209(20):6181-6189, 2009.

[58] R. Friedrich, G. Radons, T. Ditzinger, and A. Henning. Ripple formation through an interface insta- 
bility from moving growth and erosion sources. Phys. Rev. Lett., 85(23):4884-4887, 2000.

[59] G. Radons, T. Ditzinger, R. Friedrich, A. Henning, A. Kouzmichev, and E. Westkmper. Nonlinear dynamics and control of ripple formation in abrasive water-jet cutting. In Nonlinear Dynamics of Production Systems, pages 391-410. Wiley-VCH Verlag GmbH \& Co. KGaA, 2005.

[60] P. Beltrame, P. Hänggi, and U. Thiele. Depinning of three-dimensional drops from wettability defects. Europhys. Lett., 86:24006, 2009. doi:10.1209/0295-5075/86/24006.

[61] D. Tseluiko, M. Galvagno, and U. Thiele. Heteroclinic snaking near a heteroclinic chain in dragged meniscus problems. Eur. Phys. J. E, 2014. (at press). arXiv:1307.4618.

[62] J. H. Siggers. Dynamics of target patterns in low-Prandtl-number convection. J. Fluid Mech., 475:357$375,2003$.

[63] B. Krauskopf and B. E. Oldeman. A planar model system for the saddle-node Hopf bifurcation with global reinjection. Nonlinearity, 17:1119-1151, 2004. doi:10.1088/0951-7715/17/4/001. 